Article

\title{
Low-Loss Hollow Waveguide Fibers for Mid-Infrared Quantum Cascade Laser Sensing Applications
}

Pietro Patimisco ${ }^{1, *}$, Vincenzo Spagnolo ${ }^{1}$, Miriam S. Vitiello ${ }^{2,3}$, Gaetano Scamarcio ${ }^{1}$, Carlos M. Bledt ${ }^{4}$ and James A. Harrington ${ }^{4}$

1 CNR-IFN and Dipartimento Interateneo di Fisica, Università and Politecnico di Bari, Via Amendola 173, I-70126 Bari, Italy; E-Mails: vincenzo.spagnolo@uniba.it (V.S.); gaetano.scamarcio@uniba.it (G.S.)

2 CNR-Dipartimento di Scienze Fisiche e Tecnologie della Materia, Largo E. Fermi 5, 50125 Firenze, Italy; E-Mail: miriam.vitiello@sns.it

3 NEST, Istituto Nanoscienze-CNR and Scuola Normale Superiore, Piazza San Silvestro 12, I-56127 Pisa, Italy

4 Department of Material Science \& Engineering, Rutgers University, Piscataway, NJ 08855, USA; E-Mails: carlos_bledt@brown.edu (C.M.B.); jaharrin@rutgers.edu (J.A.H.)

* Author to whom correspondence should be addressed; E-Mail: pietro.patimisco@uniba.it.

Received: 19 November 2012; in revised form: 8 January 2013 / Accepted: 9 January 2013 / Published: 21 January 2013

\begin{abstract}
We report on single mode optical transmission of hollow core glass waveguides (HWG) coupled with an external cavity mid-IR quantum cascade lasers (QCLs). The QCL mode results perfectly matched to the hybrid $\mathrm{HE}_{11}$ waveguide mode and the higher losses TE-like modes have efficiently suppressed by the deposited inner dielectric coating. Optical losses down to $0.44 \mathrm{~dB} / \mathrm{m}$ and output beam divergence of $\sim 5 \mathrm{mrad}$ were measured. Using a HGW fiber with internal core size of $300 \mu \mathrm{m}$ we obtained single mode laser transmission at $10.54 \mu \mathrm{m}$ and successful employed it in a quartz enhanced photoacoustic gas sensor setup.
\end{abstract}

Keywords: hollow glass waveguide; quantum cascade laser; gas sensing; quartz enhanced photoacoustic spectroscopy 


\section{Introduction}

Fiber-optic components show enhanced versatility with respect to bulk optics, mostly in terms of flexible beam guidance and compactness. Furthermore, fibers could also be deployed advantageously as modal wavefront filters, optical path delay length control or for multi-axial beam combining [1]. There are various fields of application for infrared fibers, each one requiring special fiber properties such as: nulling interferometry [2], high precision imaging and spectroscopy [3]. Moreover, fiber optics is a key enabling technology needed to improve the robustness and effectiveness of specific detection and calibration systems.

Optical fibers can be characterized by structure, guiding-type, and material. A simple classification by structure is: solid-core fibers, hollow waveguides and micro-structured fibers (so-called photonic crystal fibers). Waveguiding in solid-core fibers obeys the principle of total reflection of light propagating inside the core. Total reflection is caused by refractive index differences between the core and cladding materials. Fibers transmitting light of wavelength larger than $2 \mu \mathrm{m}$ can be manufactured using glass or crystalline materials [4]. In this spectral range silica fibers transmission is limited by multi-phonon absorption [5].

\section{Hollow Glass Waveguides}

In hollow core glass waveguides (HGWs) light can propagate through an air core by multiple reflections on the metallic inner wall [6]. The schematic of a typical hollow guide fiber is shown in Figure 1. The main advantages are high power threshold, low insertion loss, low nonlinearity, and no end-reflection [7]. Furthermore, hollow waveguides show low beam divergence and losses are proportional to $a^{-3}$, where $a$ is the bore radius. Drawbacks are the high bending losses, which are proportional to $\mathrm{R}^{-1}$, where $\mathrm{R}$ is the bending radius [8]. Considering the cylindrical geometry of an hollow waveguide, the optical modes are transverse electric $\left(\mathrm{TE}_{l m}\right)$ or transverse magnetic $\left(\mathrm{TM}_{l m}\right)$. There are also hybrid or mixed modes designated by $\mathrm{HE}_{l m}$ and $\mathrm{EH}_{l m}$. These hybrid fiber modes have a small component of the electric field along the fiber or optic axis and correspond to skew rays (a ray that does not cross the fiber axis; rather it travels in a corkscrew or helical path down the fiber). In order to reduce the overall waveguide transmission losses due to the absorption of radiation in the metallic layer, the waveguide core is additionally coated with dielectric films [9]. Actually, the losses at IR frequencies are greatly reduced by adding dielectric thin films to metallic or metallic coated waveguides. The thickness of the dielectric film chosen to minimize losses depends on the wavelength and on the refractive index of the dielectric material employed [10]. A schematic sketch of the proposed waveguide concept is reported in Figure 1.

In a hollow waveguide the total transmission losses depend on the nature and number of the propagating modes and increase dramatically for the higher order modes [6]. Therefore, it is often desirable to excite through the waveguide only the lowest-order mode and when a Gaussian beam is focused on-axis into a hollow waveguide, only the hybrid $\mathrm{HE}_{1, \mathrm{~m}}$ modes are excited.

Characterization of mode dispersion characteristics and propagation losses is conventionally done by employing time domain spectroscopy or bulky gas lasers. However, to address the mode stability 
and to evaluate the bending loss, the characterization system is required to distinguish between waveguide modes.

In the present work we report on experimental studies aiming at coupling the low loss optical modes of hollow waveguides having a metal and dielectric internal coating with high efficiency mid-IR quantum cascade lasers (QCLs), therefore prospecting a novel integrated optical system suitable for high sensitivity and high resolution spectroscopy experiments.

Figure 1. Cross-section of the cylindrical hollow waveguides. The inner part consists of a metallic Ag layer coated by a single dielectric film of AgI or polystyrene (Ps) or by AgI/Ps double dielectric film. The outer part consists of silica with UV acrylate external coating.

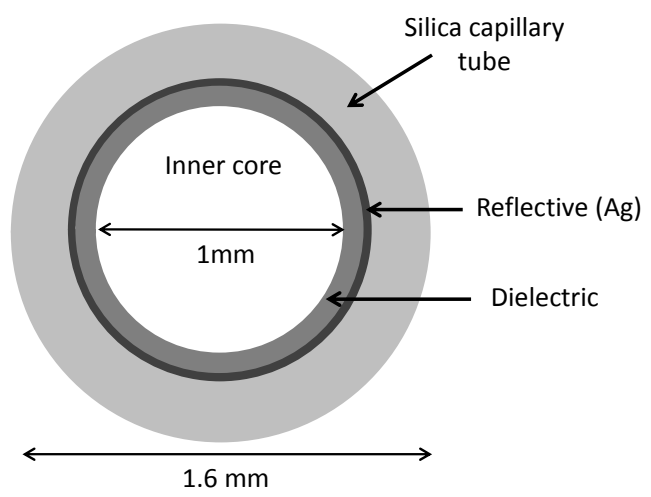

\section{Experiments and Results}

HWGs having lengths in the range 2-14 $\mathrm{cm}$ and bore diameter of $1 \mathrm{~mm}$ have been used to guide the optical beam of two external cavity edge-emitting mid-IR QCLs [11]. Three different dielectric coating were deposited over the internal silver layer with thickness of $1 \mu \mathrm{m}$ : (i) $\mathrm{AgI}(0.6 \mu \mathrm{m})$; (ii) polystyrene (Ps, $7.7 \mu \mathrm{m})$; (iii) a double dielectric layer of AgI/Ps $(0.6 / 7.7 \mu \mathrm{m})$. The fabrication of Ag/AgI-coated HGWs begins with a silica tubing, which has polymer (UV acrylate) coating on the outside surface. A liquid-phase chemistry technique [7] is used to deposit the Ag and the dielectric films inside the glass tubing. The first step involves depositing a silver film using standard Ag plating technology. Immediately after the silver is deposited, an iodine solution is pumped through the tubing and, through a subtraction process, a layer of $\mathrm{AgI}$ is formed. By controlling the concentration of the iodine solution and reaction time, an AgI film of the correct optical thickness can be deposited. To prepare silver/polystyrene (Ps)-coated hollow glass waveguides, an $\mathrm{Ag}$ film is first deposited within a glass substrate tube by combining a solution containing $\mathrm{AgNO}_{3}$ and $\mathrm{NH}_{4} \mathrm{OH}$ with a reducing solution containing dextrose and $\mathrm{Na}_{2}$ EDTA [12]. The Ag film grows at a rate of approximately $1 \mu \mathrm{m} / \mathrm{h}$, and Ag films were normally about $1 \mu \mathrm{m}$ thick. The Ps films were deposited over the Ag coatings by using a liquid-flow coating process in which a Ps/toluene solution is drawn through the HGW at a constant coating velocity.

In all cases the coating is expected to reduce the mode dispersion and the attenuation of the optical modes with lower losses. Indeed, adding a thin film on the inner surface of silver wall changes the boundary conditions and can in principle reverse the dominant mode order. As a consequence, 
the thickness for the non-absorbing coating can enhance or suppress specific modes, if the inner coating thickness is larger than the critical value [9]:

$$
d=\frac{\lambda}{2 \pi \sqrt{n^{2}-1}} \tan ^{-1}\left[\frac{n}{\sqrt[4]{n^{2}-1}}\right]
$$

where $\mathrm{n}$ is the refractive index of the dielectric film and $\lambda$ is the wavelength of propagating light in the waveguide. For what concern the thickness of PS, using Equation (1), we estimate a critical value of the layer thickness of $d_{P S}=0.53 \mu \mathrm{m}\left(\lambda_{\mathrm{a}}=5.7 \mu \mathrm{m}\right)$ and $d_{\mathrm{PS}}=1.1 \mu \mathrm{m}\left(\lambda_{\mathrm{b}}=10.5 \mu \mathrm{m}\right)$. The PC layer thickness has been set to $7.7 \mu \mathrm{m}$, much higher than the critical value for both wavelengths. We select this layer thickness since poor quality interference patterns in the near IR have been observed for thinner PC layers, meaning that the films are not uniform and so lower reflectance and thus larger losses are expected.

Two commercial mid-IR QCL sources based on an external cavity configuration were used for our experiments. The selected emission wavelengths are $\lambda_{\mathrm{a}}=5.27 \mu \mathrm{m}$ and $\lambda_{\mathrm{b}}=10.5 \mu \mathrm{m}$ (Daylight Solutions Inc., San Diego, CA, USA, model \#21052-MHF and \#21106-MHF, respectively). The average optical powers measured using a pyroelectric detector were $93 \mathrm{~mW}\left(\lambda_{\mathrm{a}}\right)$ and $65 \mathrm{~mW}\left(\lambda_{\mathrm{b}}\right)$. We tested metallic/dielectric waveguides for both wavelengths, considering that at the employed laser wavelengths, the dielectric film thickness deposited in the inner waveguide core are larger than the critical coating thickness for AgI and Ps.

The intensity profile of the propagating optical mode has been collected by means of a pyroelectric detector having a sensitive area of $1 \mathrm{~mm} \times 1 \mathrm{~mm}$, mounted on a compact motorized XY stage. The 2D multi-point image of the beam profile was obtained by recording the total transmitted power in the range 1-5.1 cm away from the waveguide output in the far field configuration for each scanning position, with a spatial resolution of about $0.2 \mu \mathrm{m}$ for both XY scanning directions.

A first set of experiments were carried out coupling the QCL and the hollow waveguide by using a $\mathrm{ZnSe}$ lens to focus the collimated beam into a $\sim 100 \mu \mathrm{m}$ spot at the waveguide entrance [13-15], as schematically shown in Figure 2(a).

Figure 2. Schematics of the experimental setup. The laser beam is focused at the waveguide entrance using a $\mathrm{ZnSe}$ lens (a) or in a simple back-to-back configuration (b) and detected using a pyroelectric detector equipped with a XY stepper motor.

a)

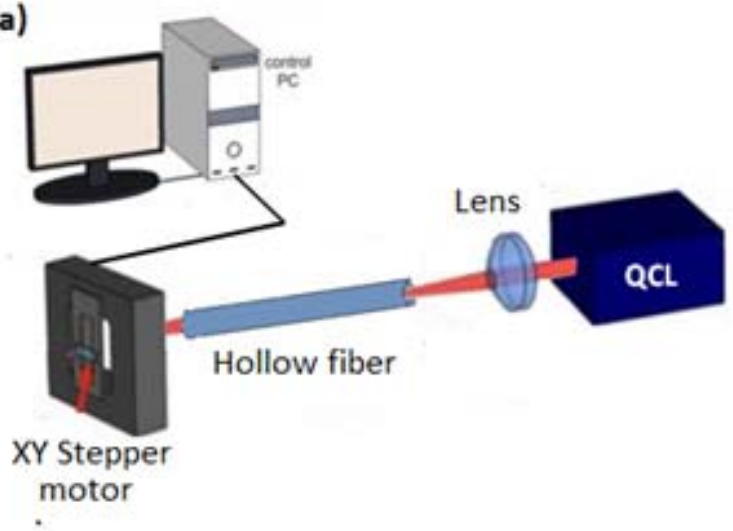

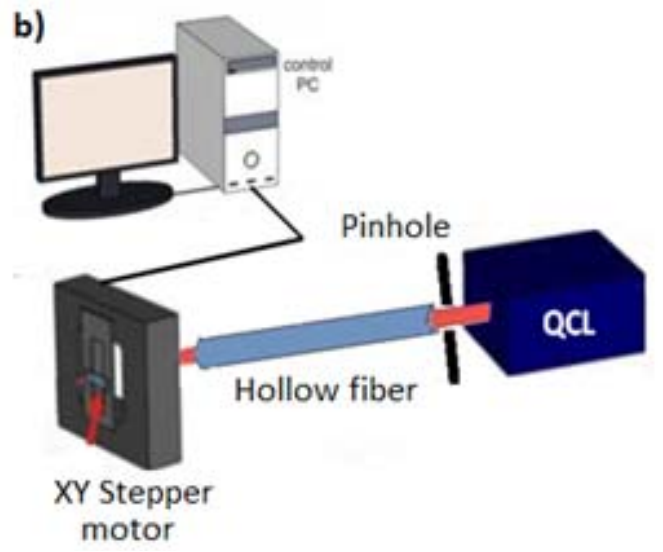


The focusing lens allows to obtain an input spot size smaller than the waveguide bore diameter in order to reduce the amount of energy blocked by the waveguide walls, thus reducing the light absorption heating, which is a critical point for high-power applications.

Figure 3 shows a representative far field 3D spatial intensity distribution measured for the $\lambda_{b}$ QCL source The QCL current was set at $900 \mathrm{~mA}$. The intensity distribution profile exhibits a Gaussian-like beam shape, as expected for a standard edge emitting QCL.

Figure 3. Far field spatial intensity distribution of the $\lambda_{b}-Q C L$. The beam profile has been measured under the experimental configuration of Figure 2(b) without any optics between the laser and the detector stage.

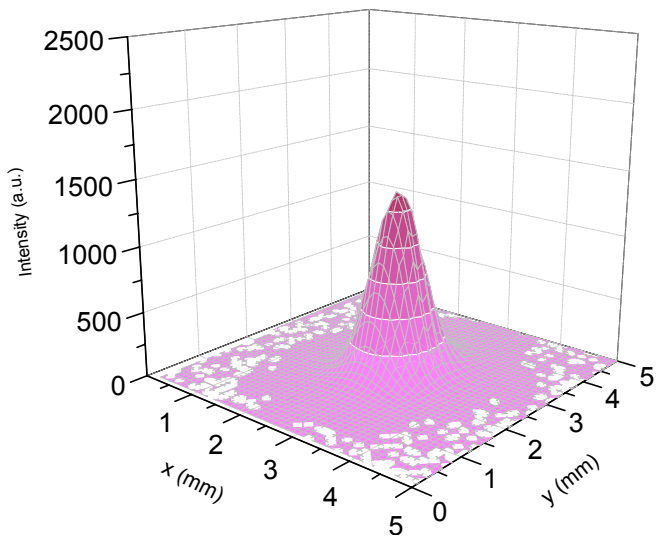

Figure 4 shows the far-field spatial intensity distribution of the two employed QCLs at the exit of a $12 \mathrm{~cm}$ long waveguide for different internal dielectric coating configuration measured $2 \mathrm{~cm}$ away from the waveguide output.

In a different experimental scheme, the QCL sources were placed directly in close contact with the fiber input with no intermediate optics, in so-called back-to-back configuration, as schematically shown in Figure 2(b). A steel circular pinhole with the same diameter of the hollow core was placed in contact with the waveguide exit to prevent detection of scattered light not propagating through the waveguide. Figure 5 shows the far-field spatial intensity distribution of the two wavelengths at the exit of a $12 \mathrm{~cm}$ long waveguide in back-to-back configuration measured $2 \mathrm{~cm}$ away from the waveguide output. The electric field intensity distribution shows that the beam shape is perfectly matched to the hybrid $\mathrm{HE}_{11}$ mode since the electric field associated to the optics field at the boundary is reduced along the entire contour of the waveguide wall, meaning that the deposited inner dielectric coating thickness suppresses effectively the higher losses TE-like modes. Note that the theoretical beam profile for $\mathrm{HE}_{11}$ mode in the waveguide can be approximated by the zero order Bessel function $E(r)=E_{0} J_{o}\left(u_{1} \frac{r}{a}\right)$.

Despite the condition a $>>\lambda$ is satisfied, we observed a single mode profile at the waveguide output in all investigated cases. However, it must be considered that in hollow waveguides the propagating losses for the higher order modes increase as the mode parameter squared $u_{\operatorname{lm}}[6]$ :

$$
\alpha_{l m}=\left(\frac{u_{l m}}{2 \pi}\right)^{2} \frac{\lambda^{2}}{a^{3}} \operatorname{Re}\left[\frac{n^{2}+1}{2 \sqrt{n^{2}-1}}\right]
$$

Thus the losses for the higher-order modes become so high that they are damped out and only the lowest-order modes can propagate. 
Figure 4. Far-Field spatial intensity distribution of the $\lambda_{a}$ (upper row) and $\lambda_{b}$ (lower row) upon exiting a $12 \mathrm{~cm}$ long and $1 \mathrm{~mm}$ core diameter hollow waveguide having AgI/Ps double dielectric layer and having a single dielectric film of AgI and Ps. The beam profiles have been measured by focusing the QCL beam directly at the center of waveguide by using a ZnSe lens.

$\operatorname{Ag} / \operatorname{AgI} / \mathbf{P s}$
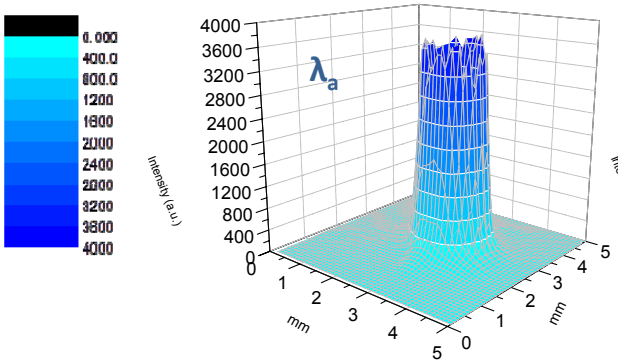

\begin{tabular}{|l}
\hline 0,002 \\
\hline 250,2 \\
530,3 \\
150,13 \\
1000 \\
4250 \\
1500 \\
1750 \\
7200 \\
2250 \\
2500 \\
\hline
\end{tabular}

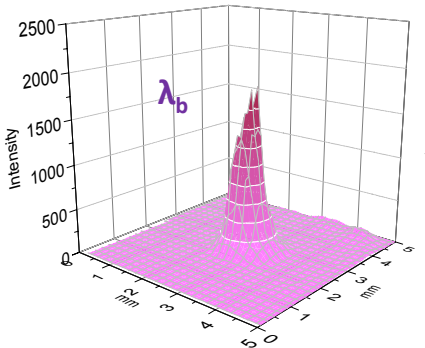

Ag/AgI
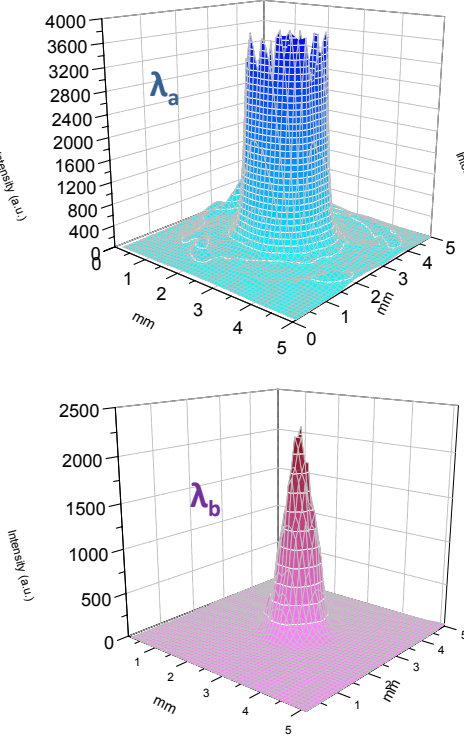

Ag/Ps
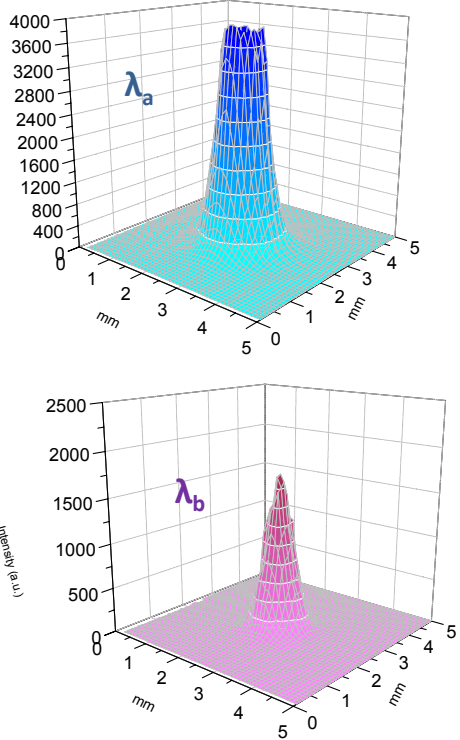

Figure 5. Far-Field spatial intensity distribution of the $\lambda_{a}$ (upper row) and $\lambda_{b}$ (lower row) upon exiting a $12 \mathrm{~cm}$ long and $1 \mathrm{~mm}$ bore diameter hollow waveguide having $\mathrm{AgI} / \mathrm{Ps}$ double dielectric layer and having a single dielectric film of AgI and Ps. The beam profiles have been measured in a back-to-back configuration.

Ag/AgI/Ps
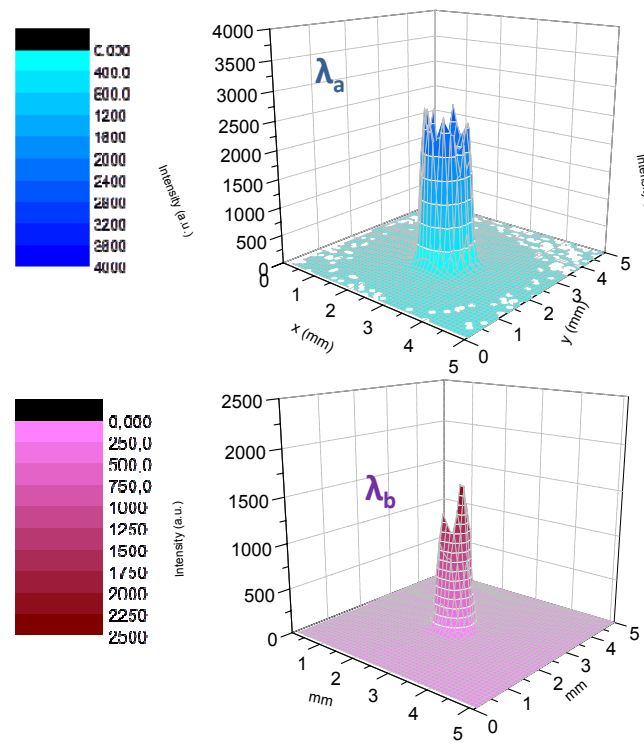

Ag/AgI
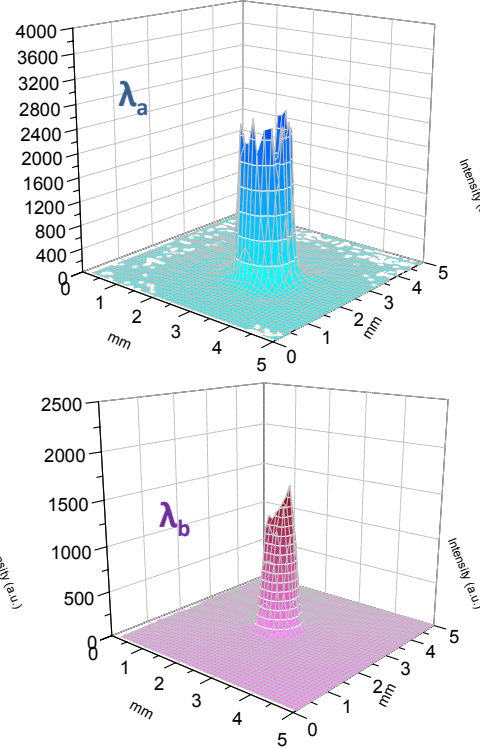

Ag/Ps
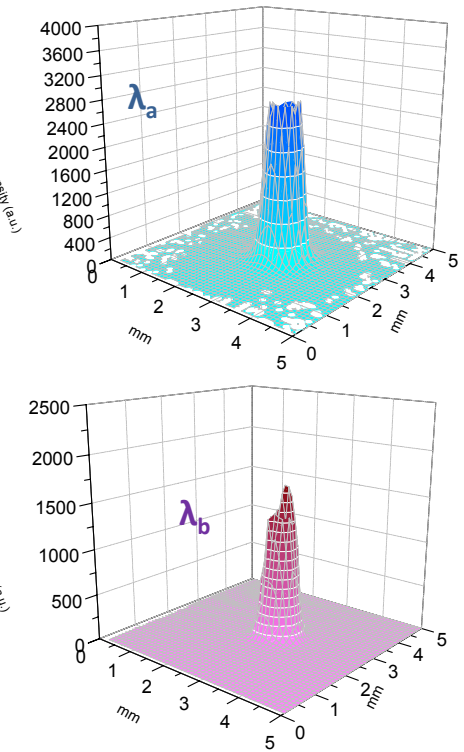
Our results demonstrate that the $\mathrm{HE}_{11}$ mode losses in an optimally designed waveguide become lower than those for $\mathrm{TE}_{01}$ mode [16], even for dielectric coatings with a moderate absorption coefficient. It is worth noting that the output beam profile of hollow waveguides depends not only on the bore diameter or the propagating wavelength, but also on the quality and launch conditions (with or without the focal lens) of the input beam, as theoretically expected [6]. We found that the beam divergence is larger when the $\mathrm{ZnSe}$ lens is used to focalize the laser beam at the waveguide entrance, especially for the wavelength $\lambda_{\mathrm{a}}$, because additional optical modes are propagating through the straight waveguide.

\section{Propagation Losses and Coupling Efficiency}

Propagating losses in hollow waveguides are highly dependent on the launch conditions, i.e., the ratio $\omega / a$, where $\omega$ is the beam waist at the waveguide entrance [8]. The power coupling efficiency of the incident beam to excite each low-loss $\mathrm{HE}_{1 \mathrm{~m}}$ waveguide mode depends critically on the beam waist to bore radius ratio. Theoretically up to $\sim 100 \%$ of power coupling efficiency of an incident Gaussian beam to the lowest-loss $\mathrm{HE}_{11}$ mode can be obtained. At small f-numbers more power is coupled to the higher-order modes. Instead at large f-numbers the beam can be clipped by waveguide walls. Since the $\mathrm{HE}_{11}$ mode has the lowest theoretical loss, it is necessary to optimize the launch conditions in order to couple most of the input beam into this mode. This is possible by changing the value of the ratio $\omega / a$ (i.e., varying the focusing lens position with respect to the waveguide entrance) and maximizing the output power in order to couple most of the light in the $\mathrm{HE}_{11}$ mode. Our experiments have been performed under these conditions.

The propagation losses and the coupling efficiency can be determined by making two measurements. First we measured the optical power I0 at the waveguide entrance, then the light is coupled into the fiber and the optical power IS at the output-end of the fiber is measured. Absolute values of the losses of the excited mode of the straight waveguide were determined by using the following equation:

$$
\mathrm{dB}=10 \log _{10}\left(\frac{\mathrm{I}_{0}}{\mathrm{I}_{\mathrm{S}}}\right)
$$

The graph in Figure 6 shows the values measured by coupling hollow waveguides of different lengths (in the range $2-14 \mathrm{~cm}$ ) with laser light at $\lambda_{\mathrm{a}}$ wavelength in back-to-back configuration.

The transmission losses increase linearly with the waveguide length. From the slope of the linear fit to the data we measured transmission losses of $0.63 \mathrm{~dB} / \mathrm{m}$ for $\mathrm{Ag} / \mathrm{AgI}$ coatings and $0.44 \mathrm{~dB} / \mathrm{m}$ for $\mathrm{Ag} / \mathrm{Ps}$ coatings. These values are nearly one order of magnitude lower that optical power attenuation measured using single-mode hollow core photonic crystal fibers made of silica glass [17].

The coupling efficiency values between the QCL source and the waveguide is extracted from the intercept at zero length of the linear fits to the data. Coupling efficiencies as high as $99.8 \pm 0.2 \%$ for the AgI dielectric film and $99.0 \pm 0.1 \%$ for Ps coating have been obtained. The small variation between the coupling efficiencies is due to slight differences in the launch conditions, i.e., the ratio $\omega / a$. The theoretical coupling efficiency is $99.6 \%$ [8]. 
Figure 6. Total losses calculated from the ratio of input/output power values for the wavelength $\lambda_{\mathrm{a}}$ at the waveguide entrance/exit for $\mathrm{Ag} / \mathrm{AgI}$ coatings ( $\bullet$ symbols) and for AgPs coatings ( symbols). The dashed lines are linear fits to the data. The reported transmission losses have been estimated from the slope of each linear fit.

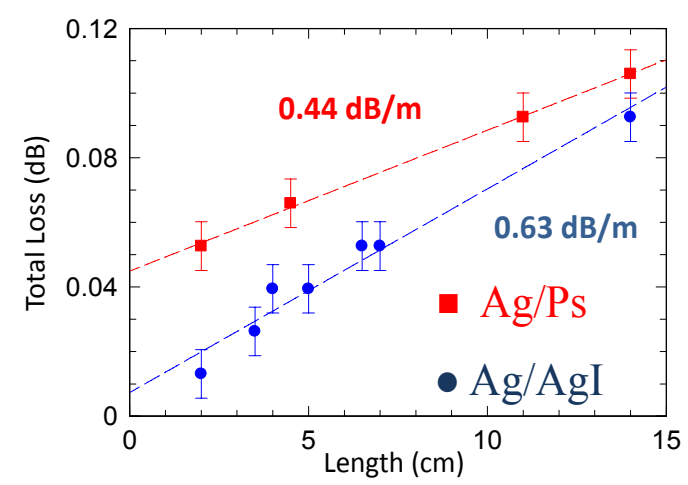

\section{Output Divergence Angle}

The output divergence angle characterizes the diffraction of light leaving the waveguide. The output-beam divergence of a HGW depends critically on three factors: (i) the nature of the optical mode propagating through the guide; (ii) the wavelength and the polarization of the incoming beam; (iii) the bore diameter.

In principle the output beam divergence can give an indication of the number of high-order mode propagating in the hollow waveguide. The $\mathrm{HE}_{1 m}$ modes will couple to free-space modes with a beam divergence $\theta_{m}$ given by:

$$
\theta_{m} \cong \sin \theta_{m}=\frac{u_{1 m} \lambda}{2 \pi a}
$$

where $u_{1 m}$ is a parameter which depends on the order number $(m)$ of the $\mathrm{HE}_{1 m}$ mode. The values for $u_{1 m}$ rapidly increase for the higher-order modes [7,9]. The schematic of the output divergence measurements is shown in Figure 7.

Figure 7. Schematics of the output divergence angle measurement.

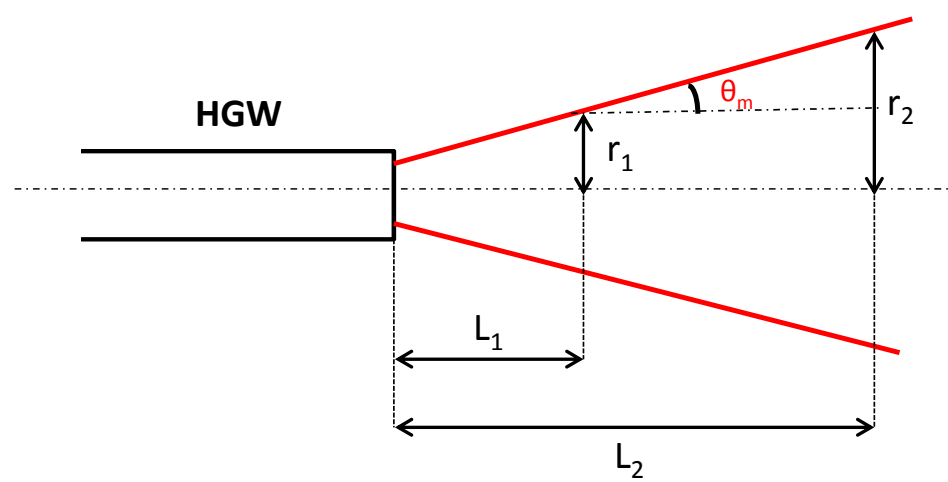

The output divergence angle $\theta_{m}$ has been calculated from:

$$
\theta_{m} \cong \tan \theta_{m}=\frac{r_{2}-r_{1}}{L_{2}-L_{1}}
$$


where $r_{1}$ and $r_{2}$ are the radial distances at which the light intensity drops to $1 / e^{2}$ of its maximum central value, measured respectively at $L_{1}=1 \mathrm{~cm}$ and $L_{2}=5.1 \mathrm{~cm}$ distance from the fiber output as measured from the far-field scans.

Figure 8 shows the 3D-far field scans measured for a $12 \mathrm{~cm}$ long HWG with AgI dielectric internal coating and at $\lambda_{\mathrm{a}}$ propagating wavelength. From these data we extracted $r_{1}=0.5 \mathrm{~mm}$ and $r_{2}=0.75 \mathrm{~mm}$ and estimated a divergence of $\theta_{m}=4.92 \mathrm{mrad}$. By using Equation (4), we estimated a mode parameter $u_{1 m}=2.94$, in good agreement with the expected theoretical value for the first propagating mode $u_{11}=2.405$.

Figure 8. Far-field spatial intensity distribution upon exiting a $12 \mathrm{~cm}$ long and $1 \mathrm{~mm}$ bore diameter hollow waveguide having a single dielectric film of $\mathrm{AgI}$ and $\mathrm{Ps}$ at $\mathrm{L}_{1}=1 \mathrm{~cm}(\mathbf{a})$ and $L_{2}=5.1 \mathrm{~cm}(\mathbf{b})$ distance from the waveguide exit.
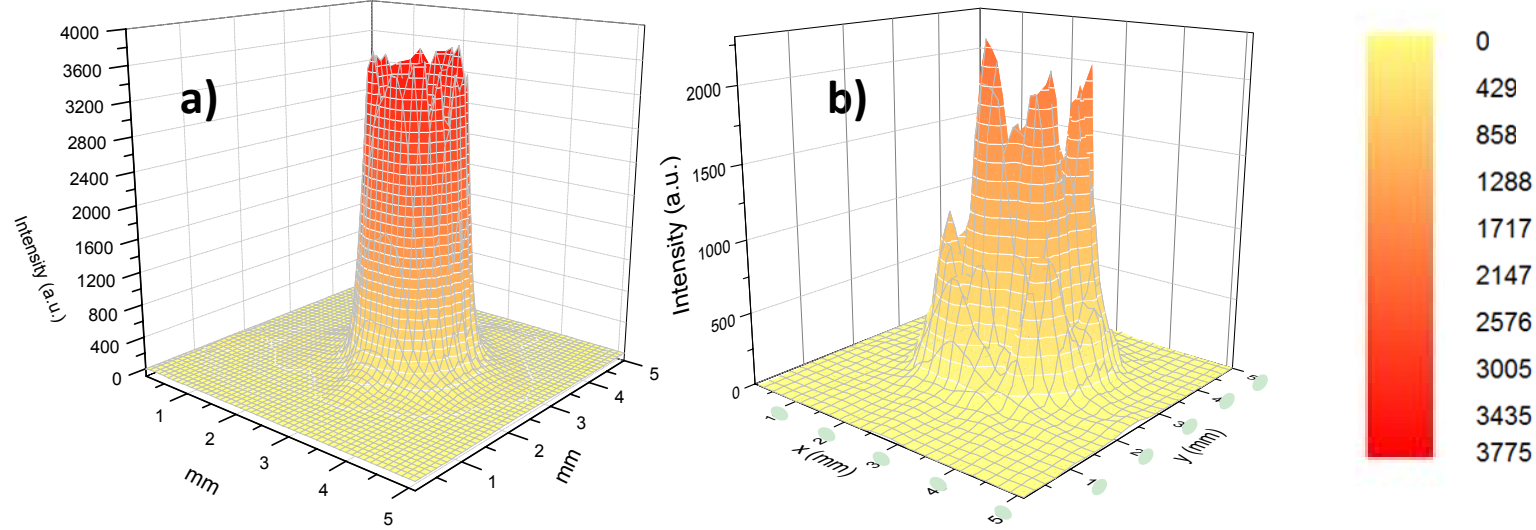

Much larger values are expected for higher order mode [6], as shown in Table 1 further confirming single-mode propagation of the laser beam in the hollow waveguide and good mode coupling between the laser-input optical mode and the waveguide lowest-loss $\mathrm{HE}_{11}$ mode.

Table 1. Values for the mode parameter $\mathrm{u}_{1 \mathrm{~m}}$ for some of the lowest-order mode.

\begin{tabular}{cc}
\hline & $\boldsymbol{l}=\mathbf{1}$ \\
\hline$m=1$ & 2.405 \\
$m=2$ & 5.52 \\
$m=3$ & 8.654 \\
$m=4$ & 11.796 \\
\hline
\end{tabular}

\section{Single-Mode HWG for Optical Sensing Applications}

A particularly attractive application for IR-fiber optics is optical sensing. In general, silica-based optical fibers are used in a large number of sensor applications such as: chemical and radiometric sensing, fiber optic gyros, etc. Fewer applications for IR-fibers have been demonstrated. The main reasons are the higher losses and the difficulty to achieve single mode propagation fibers, which are typically required in spectroscopic sensors.

For examples, in quartz enhanced photoacoustic (QEPAS) sensing, it is critical to avoid laser illumination of the acoustic detection module (ADM), since the radiation blocked by the Quartz tuning 
fork (QTF) and associated micro-resonator tubes creates an undesirable background when absorbed by the ADM structural elements. This background is usually several times larger than the thermal noise level of QEPAS and carries a shifting fringe-like interference pattern, which limits the detection sensitivity [18-20]. Thus, it is important to employ a mid-infrared QEPAS excitation beam of high quality and stability and if employing a fiber coupling system, single mode beam delivery is strictly required. Recently, we designed and assembled a QEPAS system fiber coupled with a single mode QCL and exploited its high performance for the detection of $\mathrm{SF}_{6}$ gas traces in the ppt range. Single mode laser delivery has been obtained using a hollow fiber with inner Ag-AgI coatings and an internal bore size of $300 \mu \mathrm{m}$, having a loss of $1 \mathrm{~dB} / \mathrm{m}$. Additional collimating optics have been designed to be attached to the output of the fiber and provide a focusing distance of $40 \mathrm{~mm}$. In Figure 9 is shown the 3D laser beam profile measured using a pyroelectric camera (mod. Spiricon Pyrocam IIIC, Ahrensburg Germany) at the output of the fiber (Figure 9(a)) and at the focusing plane of the collimator (Figure 9(b)). From Figure 8(b) we can estimate a focused beam waist diameter of $\sim 170 \mu \mathrm{m}$, well below the gap between the QTF prongs $(\sim 300 \mu \mathrm{m})$. As a results, in the QEPAS experiments almost all (99.4\%) of the laser beam coming out the collimator were transmitted through the ADM module without touching it, thus reducing the background pattern and allowing to reach detection sensitivity in the part-per-trillion (ppt) concentration range. A record normalized noise equivalent absorption coefficient for QEPAS of $2.7 \times 10^{-10} \mathrm{~cm}^{-1} \cdot \mathrm{W} / \mathrm{Hz}^{1 / 2}$ was achieved [20].

Figure 9. Three-dimensional single mode profile of the EC-QCL beam: exiting the hollow fiber (a) and at the focusing plane of the collimator (b). The color map, identical for both panels, is shown in right side of the figure.
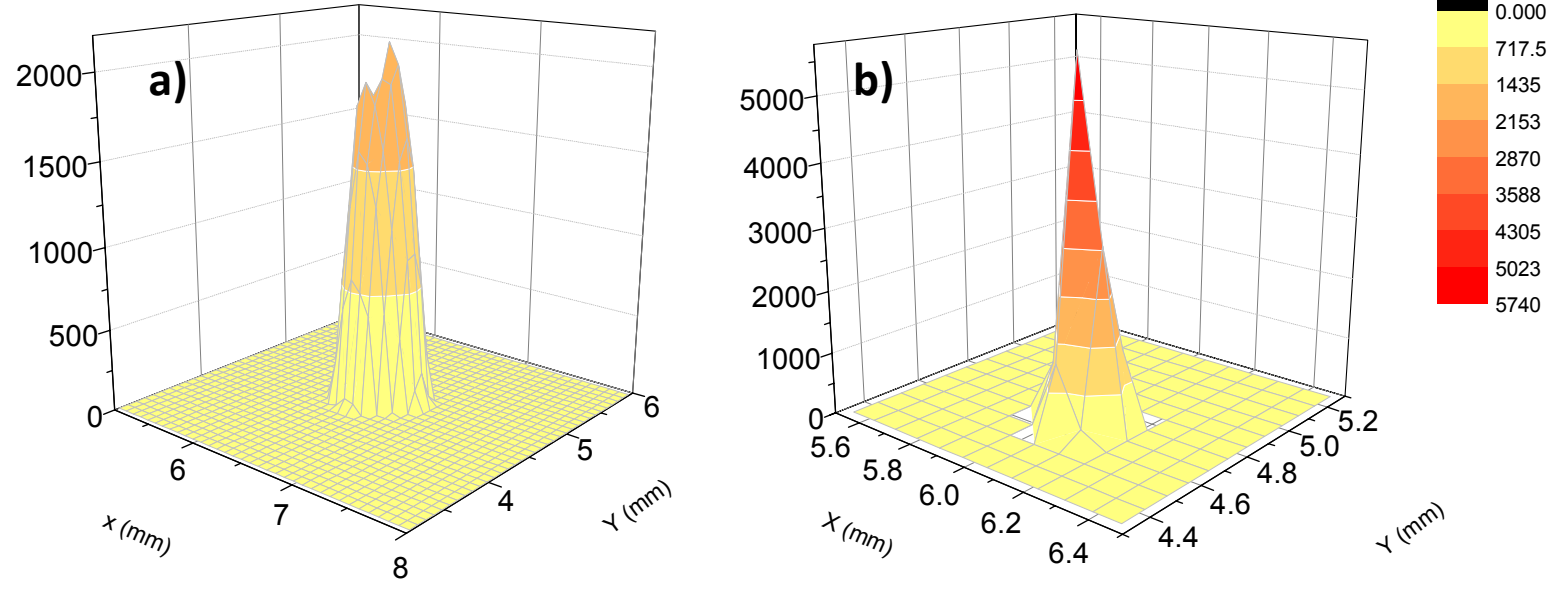

\section{Conclusions}

Optical coupling between mid-IR QCLs and metallic hollow waveguides with a single or double internal dielectric coating was investigated by mid-IR far-field imaging. Single-mode propagation of the laser beam in the hollow waveguide and excellent optical mode coupling between the incoming beam and the waveguide mode have been observed. The optical losses measured for 1,000 $\mu \mathrm{m}$ bore diameter at $5.27 \mu \mathrm{m}$ was $0.63 \mathrm{~dB} / \mathrm{m}$ for $\mathrm{Ag} / \mathrm{AgI}$ coatings and $0.44 \mathrm{~dB} / \mathrm{m}$ for $\mathrm{Ag} / \mathrm{Ps}$ coating. The output divergence angle at the $\mathrm{Ag} / \mathrm{AgI}$ waveguide exit was $<5 \mathrm{mrad}$ for $\lambda=5.27 \mu \mathrm{m}$, which makes it possible to use these fibers in several applications such as radiometry or chemical sensing. Single mode hollow 
fibers have been recently employed in a QEPAS experiments to couple the output beam of a quantum cascade laser with the acoustic detection module of the sensors. Highly focused beams were obtained, allowing an almost perfect transmission of the laser beam trough the module. This strongly reduces the sensor background signal, allowing record part-per-trillion detection limit for $\mathrm{SF}_{6}$.

\section{Acknowledgements}

The authors gratefully acknowledge partial financial support from the Italian research projects: PON01_02238 and PON02_00675.

\section{References}

1. Ksendzov, A.; Lay, O.; Martin, S.; Sanghera, J.S.; Busse, L.E.; Kim, W.H.; Pureza, P.C.; Nguyen, V.Q.; Aggarwal, I.D. Characterization of mid-infrared single mode fibers as modal filters. Appl. Opt. 2007, 46, 7957-7962.

2. Ksendzov, A.; Lewi, T.; Lay, O.P.; Martin, S.R.; Gappinger, R.O.; Lawson, P.R.; Peters, R.D.; Shalem, S.; Tsun, A.; Katzir, A. Modal filtering for midinfrared nulling interferometry using single mode silver halide fibers. Appl. Opt. 2008, 47, 5728-5735.

3. Kriesel, J.M.; Gat, N.; Bernacki, B.E.; Erikson, R.L.; Cannon, B.D.; Myers, T.L.; Bledt, C.M.; Harrington, J.A. Hollow Core Fiber Optics for Mid-Wave and Long-Wave Infrared Spectroscopy. In Proceedings of SPIE 8018, Chemical, Biological, Radiological, Nuclear, and Explosives (CBRNE) Sensing XII, Orlando, FL, USA, 25 April 2011.

4. Pinnow, D.A.; Gentile, A.L.; Standlee, A.G.; Timper, A.J.; Hobrock, L.M. Polycrystalline fiber optical waveguides for infrared transmission. Appl. Phys. Lett. 1978, 33, 28-29.

5. Spott, A.; Liu, Y.; Baehr-Jones, T.; Ilic, R.; Hochberg, M. Silicon waveguides and ring resonators at $5.5 \mu \mathrm{m}$. Appl. Phys. Lett. 2010, 97, 213501-213503.

6. Harrington, J.A. Infrared Fibers and Their Applications; SPIE: Bellingham, WA, USA, 2004.

7. Harrington, J.A. A review of IR transmitting, hollow waveguides. Fiber Integrated Opt. 2000, 19, 211-227.

8. Nubling, R.K.; Harrington, J.A. Launch conditions and mode coupling in hollow-glass waveguides. Opt. Eng. 1998, 37, 2454-2458.

9. Roshan, G.; Harrington, J.A. Infrared transmissive, hollow plastic waveguides with inner Ag-AgI coatings. Appl. Opt. 2005, 44, 6449-6455.

10. Marcantili, E.A.J.; Schmeltzer, R.A. Hollow metallic and dielectric waveguides for long distance optical transmission and lasers. Bell. Syst. Tech. J. 1964, 43, 1783-1809.

11. Patimisco, P.; Spagnolo, V.; Vitiello, M.S.; Tredicucci, A.; Scamarcio, G.; Bledt C.M.; Harrington, J.A. Coupling external mid-IR quantum cascade lasers with low loss metallic/dielectric waveguides. Appl. Phys. B 2012, 108, 255-260.

12. Bowden, B.; Harrington, J.A.; Mitrofanov, O. Silver/polystyrene-coated hollow glass waveguides for the transmission of terahertz radiation. Opt. Lett. 2007, 32, 2945-2947.

13. Vitiello, M.S.; Xu, J.H.; Kumar, M.; Beltram, F.; Tredicucci, A.; Mitrofanov, O.; Beere, H.E.; Ritchie, D.A. High efficiency coupling of terahertz micro-ring quantum cascade lasers to the low-loss optical modes of hollow metallic waveguides. Opt. Express 2011, 19, 1122-1130. 
14. Vitiello, M.S.; Xu, J.H.; Beltram, F.; Tredicucci, A.; Mitrofanov, O.; Beere, H.E.; Ritchie, D.A. Guiding a terahertz quantum cascade laser into a flexible silver-coated waveguide, J. Appl. Phys. 2011, 110, 063112:1-063112:5.

15. De Cumis, S.; Xu, U.; Bledt, C.M.; Harrington, J.A.; Tredicucci, A.; Vitiello, M.S. Flexible, low-loss waveguide designs for efficient coupling to quantum cascade lasers in the far-infrared. J. Infrared Millim. TeraHz Waves 2012, 33, 319-326.

16. Bowden, B.; Harrington, J.A.; Mitrofanov, O. Low-loss modes in hollow metallic terahertz waveguides with dielectric coatings. Appl. Phys. Lett. 2008, 93, 181104-181106.

17. Shephard, J.D.; MacPherson, W.N.; Maier, R.R.J.; Jones, J.D.C.; Hand, D.P.; Mohebbi, M.; George, A.K.; Roberts, P.J.; Knight, J.C. Single-mode mid-IR guidance in a hollow-core photonic crystal fiber, Opt. Express 2005, 13, 7139-7144.

18. Spagnolo, V.; Kosterev, A.A.; Dong, L.; Lewicki, R.; Tittel, F.K. NO trace gas sensor based on quartz enhanced photoacoustic spectroscopy and external cavity quantum cascade laser. Appl. Phys. B 2010, 100, 125-130.

19. Dong, L.; Spagnolo, V.; Lewicki, R.; Tittel, F.K. Ppb-level detection of nitric oxide using an external cavity quantum cascade laser based QEPAS sensor. Opt. Express 2011, 19, 24037-24045.

20. Spagnolo, V.; Patimisco, P.; Borri, S.; Scamarcio, G.; Bernacki, B.; Kriesel, J. Part-per-trillion level $\mathrm{SF}_{6}$ detection using a quartz enhanced photoacoustic spectroscopy based sensor with single-mode fiber-coupled quantum cascade laser excitation. Opt. Lett. 2012, 37, 460-462.

(C) 2013 by the authors; licensee MDPI, Basel, Switzerland. This article is an open access article distributed under the terms and conditions of the Creative Commons Attribution license (http://creativecommons.org/licenses/by/3.0/). 